\title{
Recent Books on Music and Politics
}

\section{COMPILED BY EUNICE SCHROEDER}

The books listed in this column address music as it relates to political expression or focus to a significant degree on relationships between individual musicians or musical communities and a governing authority. Most of the works listed were published within the previous half year. Readers are welcome to submit additional titles to schroeder@library.ucsb.edu for possible inclusion in the next issue.

Blues Music in the Sixties: A Story in Black and White. By Ulrich Adelt. New Brunswick, N.J.: Rutgers University Press, 2010. [viii, 192 p. ISBN-13 9780813547503. \$39.95.]

"Blut und Geist": Bach, Mendelssohn, und ihre Musik im Dritten Reich. By Jörg Hansen and Gerald Vogt. Eisenach: Bachhaus Eisenach, 2009. [59 p. ISBN-13 9783932257-06-3. €23.] Exhibition catalog.

Felsensprengerin, Brückenbauerin, Wegbereiterin: Die Komponistin Ethel Smyth = Rock Blaster, Bridge Builder, Road Paver: The Composer Ethel Smyth. Edited by Cornelia Bartsch, Rebecca Grotjahn, and Melanie Unseld. (Beiträge zur Kulturgeschichte der Musik, 2.) Munich: Allitera, 2010. [263 p. ISBN-13 9783869060682. \$28.]

Gewalt - Bedrohung - Krieg: Georg Friedrich Händels "Judas Maccabaeus": Interdisziplinare Studien. Edited by Dominik Höink and Jürgen Heidrich. Göttingen: V\&R Unipress, 2010. [242 p. ISBN-13 9783899717181. €39.90.]

Give 'Em Soul, Richard! Race, Radio, and Rhythm and Blues in Chicago. By Richard E. Stamz with Patrick A. Roberts. Urbana: University of Illinois Press, 2010. [xiv, 139 p. ISBN-13 9780252034985, \$60 (hbk.); 9780252076862, \$20 (pbk.).]

Gouverner l'opéra: Une histoire politique de la musique à Naples, 1767-1815. By Mélanie Traversier. (Collection de l'École français de Rome, 424.) Rome: École français de Rome, 2009. [692 p. ISBN-13 9782728308668. €58.]

Hip Hop and Inequality: Searching for the "Real" Slim Shady. By Simona J. Hill and Dave Ramsaran. Amherst, N.Y.: Cambria Press, 2009. [xi, 209 p. ISBN-13 9781604976519. \$104.99.]

Holocaust-Kompositionen als Medien der Erinnerung: Die Entwicklung eines musikwissenschaftlichen Gedächtniskonzepts. By Kerstin Sicking. (European University Studies, 36: Musicology, 259.) Frankfurt am Main: Lang, 2010. [408 p. ISBN-13 9783631587249. €62.80.]

Hot Stuff: Disco and the Remaking of American Culture. By Alice Echols. New York: W. W. Norton, 2010. [xxvi, 338 p. ISBN-13 9780393066753. \$26.95.]

A Jewish Orchestra in Nazi Germany: Musical Politics and the Berlin Jewish Culture League. By Lily E. Hirsch. Ann Arbor: University of Michigan Press, 2010. [viii, 258 p. ISBN-13 9780472117109. \$70.]

Music E Politics 4, Number 2 (Summer 2010), ISSN 1938-7687.

Article DOI: http://dx.doi.org/10.3998/mp.9460447.0004.207 
Kirill Kondrashin: His Life in Music. By Gregor Tassie. Lanham, Md.: Scarecrow Press, 2010. [xiii, 353 p. ISBN-13 9780810869745. \$65.]

Liszt: Virtuose subversif. By Bruno Moysan. (Perpetuum mobile.) Lyon: Symétrie, 2009. [296 p. ISBN$139782914373418 . € 45$.

Mark E. Smith and The Fall: Art, Music, and Politics. Edited by Michael Goddard and Benjamin Halligan. (Ashgate Popular and Folk Music Series.) Burlington, Vt.: Ashgate, 2010. [xvi, 209 p. ISBN-13 9780754668626, \$99.95 (hbk.); $9780754668671, \$ 24.95$ (pbk.).]

Mediterranean Israeli Music and the Politics of the Aesthetic. By Amy Horowitz. (Jewish Folklore and Anthropology Series.) Detroit: Wayne State University Press, 2010. [xvii, 251 p. + 1 CD. ISBN13 9780814334652. \$29.95.]

Musical ImagiNation: U.S.-Colombian Identity and the Latin Music Boom. By María Elena Cepeda. New York: New York University Press, 2010. [xii, 255 p. ISBN-13 9780814716915, \$65 (hbk.); 9780814716922, \$22 (pbk.).]

Music and Cyberliberties. By Patrick Burkart. (Music/Culture.) Middletown, Conn.: Wesleyan University Press, 2010. [xi, 180 p. ISBN-13 9780819569172, $\$ 70$ (hbk.); $9780819569189, \$ 24.95$ (pbk.).]

Music and Monumentality: Commemoration and Wonderment in Nineteenth-Century Germany. By Alexander Rehding. Oxford: Oxford University Press, 2009. [vii, 308 p. ISBN-13 9780195385380. \$49.95.]

Music and the Play of Power in the Middle East, North Africa, and Central Asia. By Laudan Nooshin. (SOAS Musicology Series.) Farnham, England: Ashgate, 2009. [xv, 340 p. ISBN-13 9780754634577. \$124.95.]

Music and the Southern Belle: From Accomplished Lady to Confederate Composer. By Candace Bailey. Carbondale: Southern Illinois University Press, 2010. [xiii, 255 p. ISBN-13 9780809329601. \$29.95.]

Music and Victorian Philanthropy: The Tonic Sol-Fa Movement. By Charles Edward McGuire. Cambridge: Cambridge University Press, 2009. [xxiii, 240 p. ISBN-13 9780521449687. \$90.]

Music, Culture, and Social Reform in the Age of Wagner. By James Garratt. Cambridge: Cambridge University Press, 2010. [xi, 292 p. ISBN-13 9780521110549. \$95.]

Musik als Form geistigen Widerstandes: Jüdische Musikerinnen und Musiker, 1933-1945: Das Beispiel Frankfurt am Main. Edited by Joachim Carlos Martini. Frankfurt am Main: Brandes \& Apsel, 2010. [2 vols. ISBN-13 9783860996225. €54.90.]

Musik und Verantwortung: Perspektiven der Musikpolitik in Deutschland. Edited by Karl Ermert. (Wolfenbütteler Akademie-Texte / Bundesakademie für Kulturelle Bildung Wolfenbüttel, 41.) Wolfenbüttel: Bundesakademie für kulturelle Bildung, 2009. [111 p. ISBN-13 9783929622416. $€ 9.25$.

Neue Linke / Neue Musik: Kulturtheorien und künstlerische Avantgarde in den 1960er und 70er Jahren. By Beate Kutschke. (Musik - Kultur - Gender, 2.) Cologne: Böhlau, 2007. [432 p. ISBN-13 9783412179069. €51.30.] 
Playing across a Divide: Israeli-Palestinian Musical Encounters. By Benjamin Brinner. Oxford: Oxford University Press, 2009. [360 p. ISBN-13 9780195175813, \$99 (hbk.); 9780195395945, \$25 (pbk.).]

Political Folk Music in America from Its Origins to Bob Dylan. By Lawrence J. Epstein. Jefferson, N.C.: McFarland, 2010. [xii, 201 p. ISBN-13 9780786448623. \$35.]

Politik mit sinnlichen Mitteln: Oper und Fest am Münchner Hof (1680-1745). By Sebastian Werr. Cologne: Böhlau, 2010. [330 p. ISBN-13 9783412205577. €42.90.]

Le prince et la musique: Les passions musicales de Louis XIV. Edited by Jean Duron. (Etudes du centre de musique baroque de Versailles.) Wavre: Mardaga, 2009. [309 p. ISBN-13 9782804700249. €29.]

Revolutionsidee und Staatskritik in Richard Wagners Schriften: Perspektiven metapolitischen Denkens. By Rüdiger Jacobs. (Wagner in der Diskussion, 4.) Würzburg: Königshausen und Neumann, 2010. [461 p. ISBN-13 9783826042805. €39.80.]

Rock and Roll in the Rocket City: The West, Identity, and Ideology in Soviet Dniepropetrovsk, 1960-1985. By Sergei I. Zhuk. Washington, D.C.: Woodrow Wilson Center Press; Baltimore: Johns Hopkins University Press, 2010. [xvii, 440 p. ISBN-13 9780801895500. \$65.]

Rock E Roll Jihad: A Muslim Rock Star's Revolution. By Salman Ahmad. New York: Free Press, 2010. [229 p. ISBN-13 9781416597674. \$24.99.]

Der russische Komponist Vjaceslav Artemov: Ein Beispiel für die politisch und gesellschaftlich bedingte Rezeption nonkonformistischer sowjetischer Komponisten. By Andreas Kloth. (Musikwissenschaft / Musikpädagogik in der Blauen Eule, 88). Essen: Die Blaue Eule, 2009. [465 p. ISBN-13 9783899242447. €39.]

Serbian and Greek Art Music: A Patch to Western Music History. Edited by Katy Romanou. Bristol, U.K.: Intellect, 2009. [214 p. ISBN-13 9781841502786. \$45.]

Shostakovich in Dialogue: Form, Imagery, and Ideas in Quartets 1-7. By Judith Kuhn. Farnham, England: Ashgate, 2010. [xv, 296 p. ISBN-13 9780754664062. \$99.95.]

Sibelius: A Composer's Life and the Awakening of Finland. By Glenda Dawn Goss. Chicago: University of Chicago Press, 2009. [xxi, 549 p. ISBN-13 9780226304779. \$55.]

Songs in Black and Lavender: Race, Sexual Politics, and Women's Music. By Eileen M. Hayes. (African American Music in Global Perspective.) Urbana: University of Illinois Press. [x, 231 p. ISBN-13 9780252035142, \$75 (hbk.); 9780252076985, \$25 (pbk.).]

Songs of Protest, Songs of Love: Popular Ballads in Eighteenth-Century Britain. By Robin Ganev. ～(Studies in Popular Culture.) Manchester: Manchester University Press, 2009. [xi, 241 p. ISBN-13 9780719078903. \$89.95.]

Sonic Warfare: Sound, Affect, and the Ecology of Fear. By Steve Goodman. (Technologies of Lived Abstraction.) Cambridge, Mass.: MIT Press, 2010. [xx, 270 p. ISBN 9780262013475. \$35.]

Talkin' 'bout a Revolution: Music and Social Change in America. By Dick Weissman. San Francisco: Backbeat Books, 2010. [400 p. ISBN 9781423442837. \$24.99.] 
Tönende Verführung: NS-Propaganda durch Filmmusik. By Andreas Pietsch. Berlin: MBV, Mensch und Buch Verlag, 2009. [389 p. ISBN-13 9783866646988. €44.]

Verkettungen und Querstände: Weberns Schüler Karl Amadeus Hartmann und Ludwig Zenk und die politischen Implikationen ihres kompositorischen Handelns vor und nach 1945. By Marie-Therese Hommes. (Forum Musikwissenschaft. 4.) Schliengen: Edition Argus, 2010. [441 p. ISBN-13 9783931264543. €49.]

Where the Dark and the Light Folks Meet: Race and the Mythology, Politics, and Business of Jazz. By Randall Sandke. (Studies in Jazz, 60.) Lanham, Md.: Scarecrow Press, 2010. [x, 277 p. ISBN-13 9780810866522, \$40.]

Working on a Dream: The Progressive Political Vision of Bruce Springsteen. By David Masciotra. New York: Continuum, 2010. [xvi, 266 p. ISBN-13 9780826425058. \$21.95.] 